\title{
Chain dynamics of poly(ethylene-alt- propylene) melts by means of coarse-grained simulations based on atomistic molecular dynamics
}

Cite as: J. Chem. Phys. 132, 024904 (2010); https://doi.org/10.1063/1.3280067

Submitted: 29 July 2009. Accepted: 08 December 2009. Published Online: 08 January 2010

R. Pérez-Aparicio, J. Colmenero, F. Alvarez, J. T. Padding, and W. J. Briels

ARTICLES YOU MAY BE INTERESTED IN

Time and length scales of polymer melts studied by coarse-grained molecular dynamics simulations

The Journal of Chemical Physics 117, 925 (2002); https://doi.org/10.1063/1.1481859

Dynamics of entangled linear polymer melts: A molecular-dynamics simulation The Journal of Chemical Physics 92, 5057 (1990); https://doi.org/10.1063/1.458541

Dissipative particle dynamics: Bridging the gap between atomistic and mesoscopic simulation The Journal of Chemical Physics 107, 4423 (1997); https://doi.org/10.1063/1.474784

The Journal

Submit Today

of Chemical Physics

The Emerging Investigators Special Collection and Awards Recognizing the excellent work of early career researchers! 


\title{
Chain dynamics of poly(ethylene-alt-propylene) melts by means of coarse-grained simulations based on atomistic molecular dynamics
}

\author{
R. Pérez-Aparicio, ${ }^{1, a)}$ J. Colmenero, ${ }^{1,2,3}$ F. Alvarez, ${ }^{1,2}$ J. T. Padding, ${ }^{4}$ and W. J. Briels ${ }^{4}$ \\ ${ }^{1}$ Departamento de Física de Materiales, UPV/EHU, Apartado 1072, 20080 San Sebastián, Spain \\ ${ }^{2}$ Centro de Física de Materiales (CSIC-UPV/EHU)-Materials Physics Center (MPC), Apartado 1072, \\ 20080 San Sebastián, Spain \\ ${ }^{3}$ Donostia International Physics Center, Paseo Manuel de Lardizabal 4, 20018 San Sebastián, Spain \\ ${ }^{4}$ Computational Biophysics, Faculty of Science and Technology, University of Twente, P.O. Box 217, \\ 7500 AE Enschede, The Netherlands
}

(Received 29 July 2009; accepted 8 December 2009; published online 8 January 2010)

\begin{abstract}
We present coarse-grained molecular dynamics simulations of poly(ethylene-alt-propylene) (PEP) melts, ranging in chain length from about $N_{e}$ (the entanglement length) to $N=6 N_{e}$. The coarse-grained parameters, potential of mean force and bare friction, were determined from fully atomistic molecular dynamics simulations carried out on a PEP cell containing 12 chains of 80 monomers each and subjected to periodic boundary conditions. These atomistic simulations were previously validated by means of extensive neutron scattering measurements. Uncrossability constrains were also introduced in the coarse-grained model to prevent unphysical bond crossing. The coarse-grained simulations were carried out at $492 \mathrm{~K}$ and focus on chain dynamics. The results obtained were analyzed in terms of Rouse coordinates and Rouse correlators. We observe deviations from Rouse behavior for all chain lengths investigated, even when the chain stiffness is incorporated in the Rouse model. These deviations become more important as the chain length increases. The general scenario emerging from the results obtained is that the deviations from Rouse-like behavior are due to correlations among the forces acting upon a chain bead, which seem to be related with the constraint of uncrossability among the chains. As consequence, nonexponentiality of the Rouse correlators and mode- and time-dependent friction are observed. It seems that, in the molecular weight explored, these effects still give not raise to reptation behavior but to a crossover regime between Rouse and reptation. On the other hand, the results obtained are in qualitative agreement with those expected from the so-called generalized Rouse models, based on memory function formalisms. (C) 2010 American Institute of Physics. [doi:10.1063/1.3280067]
\end{abstract}

\section{INTRODUCTION}

The combination of atomistic molecular dynamics (MD) simulations with neutron scattering experiments provides a very useful tool for investigating the structure and dynamics of polymer melts at local and intermolecular scales, i.e., where these systems display universal features of glassforming liquids (see Refs. 1-4 as recent representative references). At larger scales, polymers reveal unique dynamic processes, which are controlled by chain connectivity and molecular weight. These processes ultimately determine the rheological properties of polymer melts.

Due to both, the size of the macromolecular coils and the large relaxation times involved, conventional atomistic MD simulations of large-scale dynamics of long chain polymers are practically impossible with current day computer capabilities. Such simulations have been performed only for medium length chains where large-scale dynamics and rheological properties seem to be well described in terms of the simple Rouse model. ${ }^{5-9}$ In order to simulate dynamical and rheological behavior of long chains, one has to resort to coarse-grained models. Many different coarse-grained mod-

${ }^{a)}$ Electronic mail: r.perezaparicio@ehu.es. els have been reported in the literature, ${ }^{10}$ being perhaps the most popular one that of bead-spring type, where the polymer chain is represented as a sequence of beads connected by springs. ${ }^{11-14}$ Moreover, over the past years, many works have also considered different mapping procedures trying to bridge the gap between atomistic and coarse-grained descriptions of polymer melts (see e.g., Refs. 15-20). In these works several atoms or chemically identifiable groups are lumped together into one coarse particle. A model is then assumed to describe the interactions of the coarse-grained chains and the parameters in this model are adjusted until the coarse structure of the system is the same in both models, i.e., in the atomistic and the coarse model. Fitting is usually done by the iterated Boltzmann inversion. ${ }^{15}$ For static and thermodynamic properties this method works well. ${ }^{21}$ Since the coarse model is much smoother than the original atomistic model, its dynamics is usually much faster than that of the original model. This is advantageous in case one is only interested in static and thermodynamic properties, but a disadvantage in case one wants to study dynamic properties. In the latter case it is often sufficient to simply scale the unit of time in order to obtain the correct diffusion coefficient. ${ }^{21,22}$ A nice aspect of the method is that it takes into account the chemical nature of the polymer. 
The method of coarse-graining just described works well as long as the number of atoms lumped together in one coarse particle is not too large, and the coarse potential is sufficiently repulsive at short distances to prevent bond crossings. As soon as larger numbers of atoms are taken together to define one coarse particle two aspects must be taken into account, as emphasized in the work of Briels et $a l^{23-25}$ First, a simple rescaling of the unit of time will not be sufficient to obtain correct dynamical properties. In general it will be necessary to introduce friction forces and corresponding random forces. ${ }^{23}$ Second, the coarse potentials will be very soft and in particular will not be able to prevent bond crossings. In order to make sure that indeed bonds do not cross special measures must be taken. In the dissipative particle dynamics (DPD) method (Ref. 26) this is done by artificially making the coarse potentials sufficiently repulsive to make bond crossings improbable, although not completely impossible. A disadvantage of this method is that it introduces long range correlations, which are unrealistic by themselves, but also make the dynamics untrustworthy. Moreover only the slightest probability for bonds to cross will severely affect the long time dynamics of the chains.

Recently Padding and Briels ${ }^{24,25}$ have developed a model, including friction and random forces, but also preventing bond crossings without introducing the above mentioned negative side effects. In this model, the so-called "entanglement" points are introduced every time when a bond crossing is imminent. The potential energy in the two partaking bonds is taken to depend on the corresponding path lengths. Path length is defined as the length of the path going from one coarse particle via the entanglement point to its partner in the bond. The positions of the entanglement points are updated such that the energies in the bonds are minimal, which amounts to ensuring force equilibrium at the entanglement points. The positions of the coarse particles, also called blobs in this model, are updated according to Langevin dynamics. Just like in the cases mentioned above, the parameters in the model are adjusted until the coarse structure is the same in the coarse model and in the underlying atomistic model (see below). In particular this means that also in this model the chemical nature of the polymer is taken into account.

In this work, we apply this type of bottom up approach to construct a coarse-grained model of poly(ethylene-altpropylene) (PEP) melts starting from fully atomistic simulations properly validated by extensive neutron scattering measurements. The molecular weight of the atomistic simulated chains was $M_{w}=5600 \mathrm{~g} / \mathrm{mol}$ ( 80 monomers). By means of the above described method, we extended the coarse-grained simulation to systems with different molecular weights until $M_{w}=21000 \mathrm{~g} / \mathrm{mol}$, well above the entanglement mass of PEP $\left[M_{e}(492 \mathrm{~K}) \simeq 3360 \mathrm{~g} / \mathrm{mol}\right.$ Ref. 27]. The results obtained from the coarse-grained simulations are analyzed in terms of Rouse coordinates and Rouse correlators.

This paper is organized as follows. In Sec. II we describe the simulation methods, including the fully atomistic MD simulations and the coarse-grained model (technical details and construction of the force field). The simulated systems are described in Sec. III. Section IV gives the results analyzed in terms of the Rouse model. Finally, we present the discussion in Sec. V and the summary and conclusions in Sec. VI.

\section{SIMULATION METHODS}

\section{A. Fully atomistic molecular dynamics simulations}

The fully atomistic MD simulations were carried out by using the DISCOVER module from Accelrys with the condensed-phase optimized molecular potentials for atomistic simulation studies (COMPASS) force field. The COMPASS force field has been parametrized and validated using condensed-phase properties in addition to various $a b$ initio and empirical data for molecules. Therefore, this force field enables accurate and simultaneous prediction of structural, conformational, vibrational, and thermophysical properties that exist for a broad range of isolated molecules and in condensed phases, and under a wide range of conditions of temperature and pressure. ${ }^{28-34}$ The model cubic cell was built by means of the Amorphous Cell Protocol, originally proposed by Theodorou and Suter. ${ }^{35}$

We started from 12 different well equilibrated chains of 80 monomers each (14 424 atoms in total) and we constructed a cubic cell with periodic boundary conditions at $413 \mathrm{~K}$ and an initial density set to $0.79 \mathrm{~g} / \mathrm{cm}^{3}$, which is the experimental value at that temperature ${ }^{27}$ (Table I). Standard minimization procedures (Polak-Ribière conjugate gradients method) were applied to the constructed cell in order to minimize the so-obtained energy structure. Furthermore, a $N V T$ dynamics (constant number of atoms, volume, and temperature) of $1 \mathrm{~ns}$ was run to equilibrate the amorphous cell at $413 \mathrm{~K}$. The equilibrium density of the cell was achieved by running NPT dynamics (constant number of atoms, pressure, and temperature in the cell) at fixed atmospheric pressure $(\mathrm{P}=0.0001 \mathrm{GPa})$. After three runs of $1 \mathrm{~ns}$ we reached a density of $0.7948 \mathrm{~g} / \mathrm{cm}^{3}$ (close to the experimental value) that leads to a cell dimension of $5.2014 \mathrm{~nm}$ of side. Once the equilibrium density was obtained, the production simulation runs were carried out in the $N V T$ ensemble at $413 \mathrm{~K}$. As integration method, we have used the velocity-Verlet algorithm with a time step of 1 fs. For temperature control instead of a real temperature-bath coupling (i.e., Nosé-Hoover thermostat) we have followed a velocity scaling procedure but with a wide temperature window of $10 \mathrm{~K}$, where greater temperature fluctuations are allowed but the trajectory is disturbed less. In fact, we have checked in a similar polymeric system that by following this simple procedure we obtain similar results to those obtained with a $N V E$ ensemble (con-

TABLE I. Details of the simulated cells.

\begin{tabular}{cccc}
\hline \hline $\begin{array}{c}\mathrm{T} \\
(\mathrm{K})\end{array}$ & $\begin{array}{c}\rho_{\exp } \\
\left(\mathrm{g} / \mathrm{cm}^{3}\right)\end{array}$ & $\begin{array}{c}\rho_{\text {MDS }} \\
\left(\mathrm{g} / \mathrm{cm}^{3}\right)\end{array}$ & $\begin{array}{c}\text { Cell dimension } \\
(\mathrm{nm})\end{array}$ \\
\hline 492 & $\ldots$ & 0.7447 & 5.3155 \\
413 & 0.79 & 0.7948 & 5.2014 \\
350 & $\cdots$ & 0.8266 & 5.1338 \\
300 & 0.856 & 0.8549 & 5.0766 \\
\hline \hline
\end{tabular}




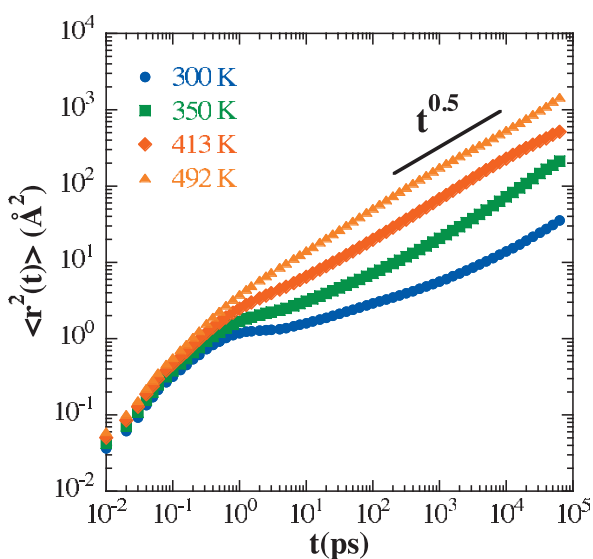

FIG. 1. Mean square displacement of the fully atomistic systems at the different simulated temperatures.

stant total energy instead of temperature), which has the proper Newtonian dynamics. First of all, an additional $1 \mathrm{~ns}$ equilibrium run was carried out without recording the trajectories of the atoms. The system so obtained was used as a starting point for collecting data during successive MD runs of 1,2 , and $100 \mathrm{~ns}$. Data were collected every 0.01, 0.05, and $0.5 \mathrm{ps}$, respectively. Finally, a further run of $1 \mathrm{~ns}$ was executed in order to check the possible appearance of aging process but nearly indistinguishable results were obtained from the consecutive simulation runs, confirming equilibration of the sample.

After the $413 \mathrm{~K}$ simulations, the cell was used to generate corresponding cells at other temperatures, namely, 492, 350 , and $300 \mathrm{~K}$. In order to do this, $N P T$ simulation runs of some nanoseconds (depending on the temperature) were used to readapt the system to each new temperature, allowing the size of the system to rearrange itself in order to accommodate to the new density at each temperature. In this way, we got the values displayed in Table I for the cell sizes and densities. Subsequent $N V T$ runs of $1 \mathrm{~ns}$ were performed for equilibration at each temperature before collecting data for analysis, in the same way as above described for $413 \mathrm{~K}$.

The results from these fully atomistic simulations have been carefully validated by comparison with experimental results from neutron scattering in the similar dynamic range on PEP samples. The static and dynamic structure factors both coherent and incoherent were calculated from the atomic trajectories obtained during the simulation runs, and directly compared with neutron scattering measurements carried out on both protonated and deuterated PEP samples, in particular, diffraction with polarization analysis and neutron spin echo measurements. A good agreement was found. The results from this comparison are beyond the aim of this paper and are reported elsewhere. ${ }^{36}$

Although in this paper we will only focus on the results corresponding to the high temperature $492 \mathrm{~K}$, Fig. 1 shows as an example the mean square displacement $\left\langle r^{2}(t)\right\rangle$ of hydrogen atoms at different temperatures. At $492 \mathrm{~K}$ and for times longer than about $2 \mathrm{ps},\left\langle r^{2}(t)\right\rangle \sim t^{0.5}$, which is the expected behavior for Rouse-like dynamics. Moreover, the figure shows that, at this temperature, there is an almost direct crossover from the microscopic regime towards the Rouse-like behavior. Only at lower temperatures a decaging range $\left(\alpha\right.$-relaxation) becomes evident before the $\left\langle r^{2}(t)\right\rangle$ $\sim t^{0.5}$ regime.

\section{B. Coarse-grained simulations}

The details of a polymer are not important in the study of the large scale dynamics, thus we can described the polymer chain in terms of groups of monomers, from now called blobs. The position $\mathbf{R}$ of each blob is defined as the center of mass position of the $\lambda$ monomers, which together constitute the blob,

$$
\mathbf{R}=\frac{1}{M} \sum_{i=1}^{\lambda} m_{i} \mathbf{r}_{i},
$$

where $\mathbf{r}_{i}$ is the position, $m_{i}$ is the mass of monomer $i$, and $M$ is the total mass of the blob. The value of $\lambda$ may be chosen arbitrarily because the interaction model is not fixed a priori, but derived without any adjustable parameters from atomistic simulations of the sample. However clearly, the number of monomers per blob is not completely arbitrary. It should not be so large that the size of the blob exceeds the typical diameter of the tube in the reptation picture. On the other hand, it must be as large as possible to allow for a large integration step and furthermore, it must be large enough to be able to treat the complementary $3(\lambda-1)$ coordinates per blob of the microscopic constituents as bath variables, i.e., to take their effects into account through friction and random forces, which perturb the time evolution of the blob positions. If the random forces decorrelate much faster than the blob momenta, they may be represented by delta functions (Markov approximation) and the equations of motion are of the simplest Langevin type,

$$
M \frac{d^{2} \mathbf{R}_{i}}{d t^{2}}=-\nabla_{i} \chi-\zeta \frac{d \mathbf{R}_{i}}{d t}+\mathbf{F}_{i}^{R},
$$

where $\chi$ is the potential of mean force of the blob system and $\zeta$ is the blob friction coefficient. The friction on each blob is chosen to be isotropic and independent of the positions of the other blobs, in which case the friction is a scalar quantity. It is also related to the random force $\mathbf{F}^{R}$ through the fluctuation dissipation theorem,

$$
\left\langle\mathbf{F}_{i}^{R}(t) \cdot \mathbf{F}_{j}^{R}(0)\right\rangle=6 k_{B} T \zeta \delta_{i j} \delta(t),
$$

where $k_{B}$ is Boltzmann's constant and $T$ is the temperature.

The free energy $\chi$, which is the potential of mean force, is defined as

$$
\chi\left(\mathbf{R}^{n}\right)=-k_{B} T \ln P_{n}\left(\mathbf{R}^{n}\right) .
$$

Here $P_{n}$ is the $n$-blob distribution function, which is determined from the atomistic simulations of the microscopic system by averaging over the bath variables. The occurrence of $\chi$ in the Langevin equation guarantees that the blob distributions in the coarse-grained and microscopic systems are the same. In order to calculate the distribution function we have to assume that it can be factorized into independent nonbonded, bonded, and angular parts according to 


$$
P_{n}\left(\mathbf{R}^{n}\right)=\prod_{i<j} P^{n b}\left(R_{i, j}\right) \prod_{i} P^{b}\left(R_{i, i+1}\right) \prod_{i} P^{\theta}\left(\theta_{i}\right),
$$

where $\mathbf{R}_{i, j}=\mathbf{R}_{i}-\mathbf{R}_{j}, R_{i, j}=\left|\mathbf{R}_{i, j}\right|$, and $\theta_{i}$ is the angle between two consecutive bonds. Thus, the potential of mean force is approximated as a sum of nonbonded, bonded, and angular energies,

$$
\chi\left(\mathbf{R}^{n}\right)=\sum_{i<j} \varphi^{n b}\left(R_{i, j}\right)+\sum_{i} \varphi^{b}\left(R_{i, i+1}\right)+\sum_{i} \varphi^{\theta}\left(\theta_{i}\right) .
$$

This supposition seems to be rather crude but it does not have a big impact on the long time dynamics of the simulated polymer, where the properties are dominated not so much by the details of the interactions but by the fact that chains cannot cross each other.

An important consequence of averaging out the bath variables is that the resulting bonded and nonbonded interactions become softer and broaden their range. Consequently, unphysical bond crossing could be probable. For this reason, Padding and Briels developed an algorithm, called Twentanglement, that explicitly introduces the uncrossability constraint into this type of mesoscopic simulations in order to detect and prevent unphysical bond crossing. ${ }^{24}$ This algorithm makes the bonds as slippery elastic bands between the bonded particles. As soon as two of these elastic bands make contact a so-called "entanglement" point $\mathbf{X}$ is created, and as the blobs continue to move, the entanglement point shifts, such that it will push both bonds back to their respective sides. This is accomplished by defining the attractive part of the potential $\varphi^{\text {att }}$ between bonded blobs $i$ and $i+1$ to be a function of the path length $L_{i, i+1}$ of the bond, going from one blob $(i)$ to the next $(i+1)$ via the "intermediate entanglement,"

$$
\begin{aligned}
& L_{i, i+1}=\left|\mathbf{R}_{i}-\mathbf{X}\right|+\left|\mathbf{X}-\mathbf{R}_{i+1}\right|, \\
& \varphi^{\mathrm{att}}\left(L_{i, i+1}\right)=c_{3}\left(L_{i, i+1}\right)^{\mu},
\end{aligned}
$$

where $c_{3}$ and $\mu$ are fitting parameters.

The entanglement position $\mathbf{X}$ is determined by the requirement that there is always an equilibrium of forces at the entanglement. In a sense, the original bonds are replaced by slippery elastic bands, which go via the entanglements. The finite extensibility of the bands prevents entangled chains from crossing each other. The expression for the path length given here is only valid in case of just one entanglement between two pairs of bonded blobs, but the algorithm allows for any number of entanglements between pairs of bonded blobs. To this end, the path length concept has been trivially modified. The replacement of blob distances by path lengths in the bonded part of the potential energy changes the structural properties of the model. However, the mesoscopic distribution functions obtained by this method are hardly different from the ones obtained from the microscopic simulation.

We have to emphasize that in this code "entanglements" are defined as the objects that prevent the crossing of chains and not in the usual sense of long-lasting obstacles, slowing down the chain movement. Thereby, we can expect "entanglement effects" of this type even in simulation of rather short chains.
TABLE II. Parameters for the potentials of mean force obtained from fits of the distribution functions, as explained in the text.

\begin{tabular}{llc}
\hline \hline Parameter & Value & Units \\
\hline$c_{0}$ (nonbonded) & 1.222 & $\mathrm{~kJ} \mathrm{~mol}^{-1}$ \\
$b_{0}$ (bonded) & 0.95 & $\mathrm{~nm}$ \\
$c_{1}$ (bo & 1.02 & $\mathrm{~kJ} \mathrm{~mol}^{-1}$ \\
$b_{1}$ & 0.70 & $\mathrm{~nm}$ \\
$c_{2}$ & 3.395 & $\mathrm{~kJ} \mathrm{~mol}^{-1}$ \\
$b_{2}$ & 0.25 & $\mathrm{~nm}^{-\mu}$ \\
$c_{3}$ & 0.0265 & $\mathrm{~kJ} \mathrm{~mol}^{-1} \mathrm{~nm}^{-\mu}$ \\
$\mu$ & 3.75 & \\
$c_{4}$ (angular) & 0.397 & $\mathrm{~kJ} \mathrm{~mol}^{-1}$ \\
$\nu$ & 1.18 & \\
\hline \hline
\end{tabular}

\section{Determining the potential of mean force}

The potential of mean force [Eq. (4)] was determined from the fully atomistic simulations at $492 \mathrm{~K}$ out of the spatial distribution of blobs. Following the considerations explained in the previous section, the level of coarse graining was chosen to be ten monomers as a blob, which is one-half of the reported entanglement length. ${ }^{27}$ Thus, from the atomistic simulations (described in Sec. II A), we calculate the center of mass every ten monomers producing a system of 12 chains of eight blobs per chain. Now the relevant parameter is the center of mass of each blob, and the rest of the coordinates per blob are treated as bath variables. Thereby, we calculate the distribution functions of blobs and afterwards, by means of Eq. (4) we obtain the potential of mean force, which is finally fitted by analytical functions. The nonbonded interaction is described by a single repulsive Gaussian pair potential and the bonded interaction by a repulsive term, described by two Gaussians, and an attractive term, described by a power law. The angular potential was described as a function of the cosine of the angle between three blobs,

$$
\begin{aligned}
& \varphi^{n b}(R)=c_{0} e^{-\left(R / b_{0}\right)^{2},} \\
& \varphi^{b}(R)=c_{1} e^{-\left(R / b_{1}\right)^{2}}+c_{2} e^{-\left(R / b_{2}\right)^{2}}+c_{3}(R)^{\mu}, \\
& \varphi^{\theta}(\theta)=c_{4}(1+\cos \theta)^{\nu} .
\end{aligned}
$$

Fitting the parameters $c_{0}$ to $c_{4}, b_{0}$ to $b_{2}, \mu$, and $\nu$ (Table II) we construct the potential of mean force and we run mesoscopic simulations with the uncrossability algorithm above described in a cell of 100 chains of eight blobs (size-cell conditions explained below) with the same density and temperature of the microscopic system. Then, we calculate the distribution functions from those mesoscopic simulations and we compare them with the microscopic ones. We slightly change the parameters of the potentials and repeat the simulations until we reach an overlapping between both distribution functions (Fig. 2).

\section{Determining the bare friction coefficient}

So far, we have fixed the potential of mean force and we can describe the dynamics of blob chains in terms of the Langevin equation [Eq. (2)]. Nevertheless, only the structural properties are matched and the system has been coarse 

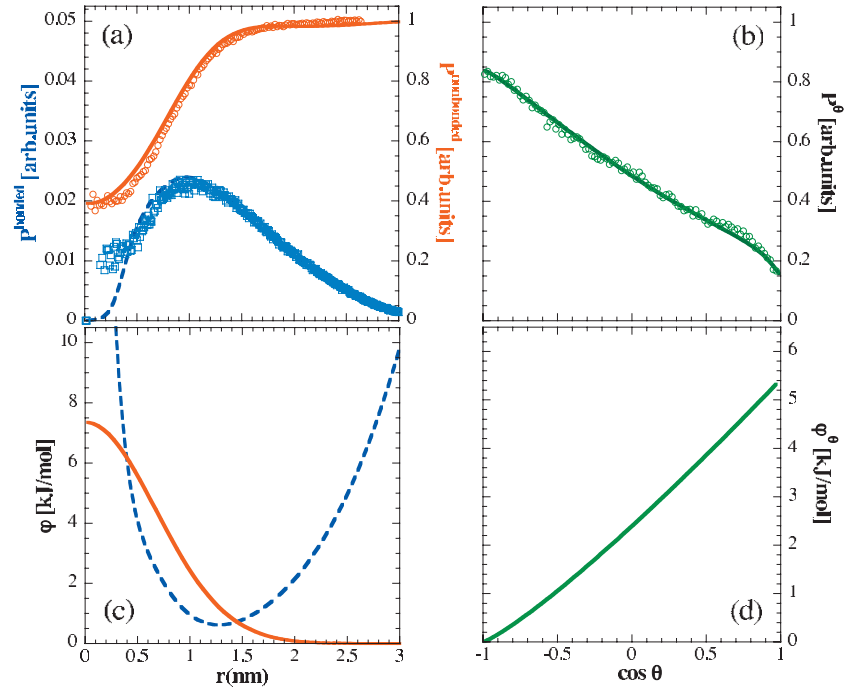

FIG. 2. Distribution functions and partial potentials of mean force. (a) shows the distribution functions from microscopic simulations (symbols) of nonbonded (circles) and bonded (squares) center of mass of ten monomer units. (b) presents the angular distribution (symbols) of the same system. Taking minus $k_{B} T$ times the logarithm of the distribution functions, partial potentials of mean force are obtained as explained in the text: in (c), nonbonded (solid line) and bonded (dashed line) potentials and in (d), angular potential (solid line). Mesoscopic simulations with these potentials and the uncrossability constraint yield the distributions given by the lines in (a) and (b).

grained to a much higher level. Thus, the interactions have become very soft and we have to adjust the blob friction coefficient $\zeta$ in order to obtain correct dynamic properties. As a consequence, besides acting as a thermostat, the friction coefficient has acquired the meaning of a "physical" friction. In this work, the blob friction coefficient was chosen such that the mean square displacement of the center of mass of the chains matched the long time behavior of that obtained from the atomistic simulations (Fig. 3). The value deduced for the friction frequency was $\zeta / M=36.0 \mathrm{ps}^{-1}$. Notice that this is the bare friction coefficient, which goes into the Langevin equation and is not necessarily related to the diffusion coefficient $D$ of the chains by $\xi=k_{B} T(N D)^{-1}$ with $N$ $=8$ like it would have been for a melt of Rouse chains. Figure 3 shows that with this value of the friction frequency, the mesoscopic coarse-grained simulations are only realistic for times longer than about 3 ns.

\section{SIMULATED SYSTEMS}

Once everything is fixed in the coarse-grained model, we constructed the initial configurations with chains of $5,8,14$, 20 , and 30 blobs randomly distributed in the simulation boxes with the microscopic density $\left(0.7447 \mathrm{~g} / \mathrm{cm}^{3}\right.$ at $492 \mathrm{~K}$, Table I). The details of the simulated systems are given in Table III. The number of chains in each system was chosen such that the length of the simulation box was at least as long as the root mean square end-to-end distance of a polymer chain in order to avoid interactions of a chain with its periodic images. ${ }^{24,25,37}$

From the initial configurations we obtained well equilibrated systems running 50 ns without the uncrossability algorithm. This run was long enough to relax the systems due

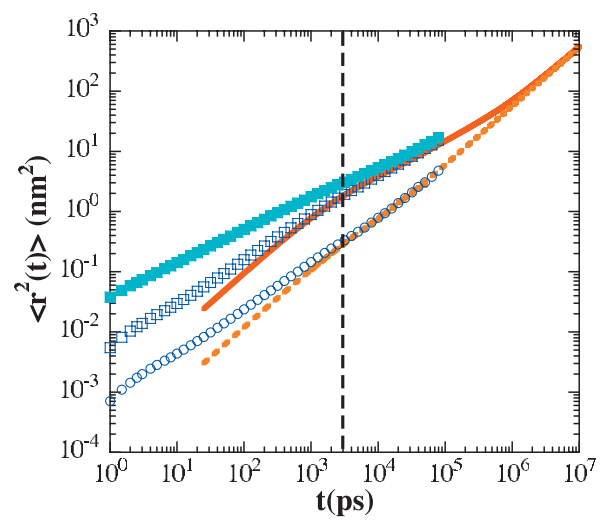

FIG. 3. Mean square displacements of entangled mesoscopic chains (lines) compared with the results of atomistic MD simulations (symbols). Blob mean square displacements are indicated by empty squares, chain center of mass mean square displacements by empty circles. The full squares correspond to the hydrogen motions of the microscopic system and the vertical line indicates the realistic limit of the coarse-grained simulations.

to the softness of the interactions by allowing all bonds to cross each other. Afterwards another run of 50 ns with uncrossability conditions was done to reach an equilibrium value of the number of "entanglements" for all systems. From this point we started collecting data. In these simulations the integration of the Langevin equation [Eq. (2)] was made using the algorithm of Allen, ${ }^{38}$ and a time step of 0.5 ps was chosen, being small enough to accurately integrate the equations of motions. The entanglement mass of PEP at $492 \mathrm{~K}$ was estimated to be $M_{e} \simeq 3360 \mathrm{~g} / \mathrm{mol}$ from the experimental values published in Ref. 27. As can be seen in Table III, the average mean square end-to-end distance and the average mean square radius of gyration of the longest chains approximately obey random walk statistics, i.e., $\left\langle R_{e e}^{2}\right\rangle /\left\langle R_{g}^{2}\right\rangle=6$.

\section{RESULTS}

Nowadays it is generally accepted that in some time regimes, the dynamics of polymer melts of medium length chains can be well approximated by the simple Rouse model. ${ }^{39,40}$ It is thereby interesting to describe our results in terms of the so-called Rouse modes (normal coordinates) and Rouse correlations (autocorrelation functions of the Rouse modes). The Rouse modes are defined as

$$
\mathbf{X}_{p}(t)=\frac{1}{N} \sum_{i=1}^{N} \mathbf{R}_{i}(t) \cos \left[\frac{p \pi}{N}\left(i-\frac{1}{2}\right)\right],
$$

where $N$ is the number of beads in the chain, $p$ is the mode number $(p=0,1,2,3, \ldots, N-1)$, and $\mathbf{R}_{i}$ is the position of bead $i$. The zeroth mode gives the position of the center of mass of the chain and the others are associated with internal motions of the chain with a "wavelength" of the order of $N / p$. Within the Rouse model, each of the modes $(p>0)$ relaxes independently and exponentially,

$$
\left\langle\mathbf{X}_{p}(t) \cdot \mathbf{X}_{p}(0)\right\rangle=\left\langle X_{p}^{2}(0)\right\rangle \exp \left(-t / \tau_{p}\right),
$$

with 
TABLE III. Simulated systems (number of blobs per chain B?), number of chains $n_{\text {chain, }}$, length $L$ of the simulation box, simulated time $t$, molecular weight of the chains, average mean square radius of gyration, average mean square end-to-end distance, and the ratio between both.

\begin{tabular}{lccccccc}
\hline \hline System & $n_{\text {chain }}$ & $\begin{array}{c}L \\
(\mathrm{~nm})\end{array}$ & $\begin{array}{c}t \\
(\mu \mathrm{s})\end{array}$ & $\begin{array}{c}M_{w} \\
(\mathrm{~g} / \mathrm{mol})\end{array}$ & $\begin{array}{c}\left\langle R_{g}^{2}\right\rangle \\
\left(\mathrm{nm}^{2}\right)\end{array}$ & $\begin{array}{c}\left\langle R_{e e}^{2}\right\rangle \\
\left(\mathrm{nm}^{2}\right)\end{array}$ & $\left\langle R_{e e}^{2}\right\rangle /\left\langle R_{g}^{2}\right\rangle$ \\
\hline B5 & 150 & 10.54 & 100 & 3500 & 3.94 & 21.79 & 5.53 \\
B8 & 100 & 10.77 & 75 & 5600 & 6.84 & 39.75 & 5.81 \\
B14 & 100 & 12.98 & 25 & 9800 & 12.79 & 76.29 & 5.96 \\
B20 & 80 & 13.57 & 18 & 14000 & 18.54 & 109.82 & 5.92 \\
B30 & 80 & 15.53 & 8 & 21000 & 28.04 & 168.20 & 5.99 \\
\hline \hline
\end{tabular}

$$
\tau_{p}^{-1}=4 W \sin ^{2}\left(\frac{p \pi}{2 N}\right)
$$

where $W$ is a characteristic frequency, the Rouse frequency, given by

$$
W=\frac{3 k_{B} T}{\xi b^{2}} .
$$

$b$ is the so-called statistical segment (the "size" of the bead) and $\xi$ is a constant friction coefficient. On the other hand, the amplitudes $\left\langle X_{p}^{2}(0)\right\rangle$ are given by

$$
\left\langle X_{p}^{2}(0)\right\rangle=\frac{b^{2}}{8 N} \sin ^{-2}\left(\frac{\pi p}{2 N}\right) .
$$

It is noteworthy that since the Rouse model does not contain an inherent length scale, the parameters $N$ and $b^{2}$ are somewhat arbitrary as long as the physical values of $b^{2} / \xi$ and $\left\langle R_{e e}^{2}\right\rangle$ are kept constant. Thereby it is easy to show from the above equations that $b^{2} / \xi$ does not depend on the level of coarse graining of the chain.

From the blob trajectories obtained in the simulations, we have calculated the Rouse modes and the Rouse correlators. Figure 4 shows as an example the normalized Rouse correlators for the system B8.

In order to check the exponentiality of the Rouse correlators, we have fitted the correlation functions by means of the well-known stretched exponential function (KohlrauschWilliams-Watts function),

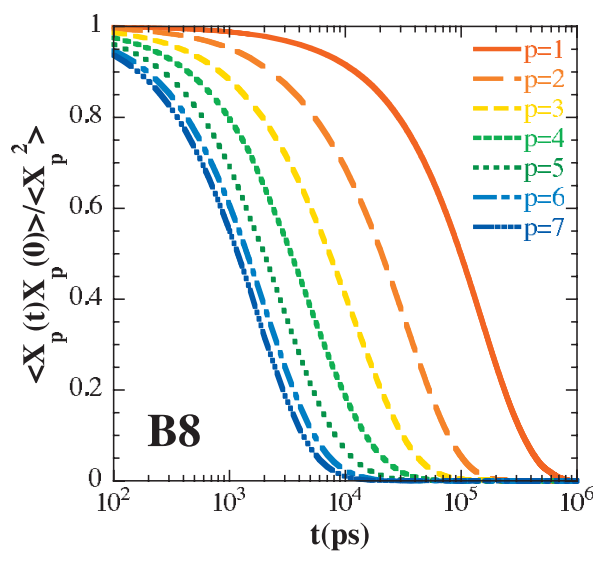

FIG. 4. Normalized Rouse correlators for the system B8.

$$
\left\langle\mathbf{X}_{p}(t) \cdot \mathbf{X}_{p}(0)\right\rangle /\left\langle\mathbf{X}_{p}^{2}(0)\right\rangle=\exp \left[\left(-t / \tau_{p}^{\mathrm{KWW}}\right)^{\beta_{p}}\right],
$$

where the relaxation time $\tau_{p}^{\mathrm{KWW}}$ and the stretching parameter $\beta_{p}$-measuring the deviations from an exponential decaydepend on mode number $p$ and, in principle, on chain length as well. The values of $\beta_{p}$ obtained for all simulated systems are shown in Fig. 5(a) as a function of the scaling variable $N / p$. Only for long wavelengths $N / p$ (low $p$-values) the stretching parameter has values close to one, confirming an almost exponential behavior. For $N / p \lesssim 10 \beta_{p}$ decreases, reaching a minimum value between $N / p \sim 2$ and $N / p \sim 3$ and then increases again. Although this trend is the same for all chain lengths, Fig. 5(a) shows that the absolute values of $\beta_{p}$ depend on $N$-at least for $N \leq 14$-being smaller as $N$ increases. A similar behavior was found in the case of coarse-grained simulations of polyethylene melts by following the same methodology as here. ${ }^{25}$ However, in that case the $N$-dependence of $\beta_{p}$ was not so evident. Nonexponential relaxation of Rouse modes has also been reported for other systems and different simulation methods as well, for example, simulations of polymers on a lattice with uncrossability constraints, ${ }^{41}$ atomistic MD simulations of polyethylene and polybutadiene (see e.g., Refs. 6 and 42), and more recently poly(ethylene oxide). ${ }^{8}$ These type of deviations have also been reported from theoretical treatments as well (see e.g., Refs. 43 and 44). Figure 5(b) shows the relaxation times of the Rouse modes for all simulated systems and also as a function of $N / p$. As $\beta_{p}$ changes for the different modes, we have represented the average relaxation times $\left\langle\tau_{p}\right\rangle$, which takes into account the shape of the relaxation function, and that for a stretched exponential is given by

$$
\left\langle\tau_{p}\right\rangle=\frac{\tau_{p}^{\mathrm{KWW}}}{\beta_{p}} \Gamma\left(1 / \beta_{p}\right),
$$

where $\Gamma$ is the Gamma function.

First of all, it is noteworthy that, according to Fig. 3, the coarse-grained simulations are not realistic at times shorter than about 3 ns. Therefore, values of $\left\langle\tau_{p}\right\rangle$ lower that this limit should not be taken into account. Figure 5(b) shows that $\left\langle\tau_{p}\right\rangle \sim 3$ ns defines a corresponding $N / p$-value below which magnitudes other than $\left\langle\tau_{p}\right\rangle$, deduced by fitting the Rouse correlators, should not be considered either. Obviously, the limitation in time scale also translates to the other magnitudes deduced by fitting the Rouse correlators.

In the framework of the Rouse model [Eqs. (13) and (14)] $\left\langle\tau_{p}\right\rangle$ values corresponding to different chains should 


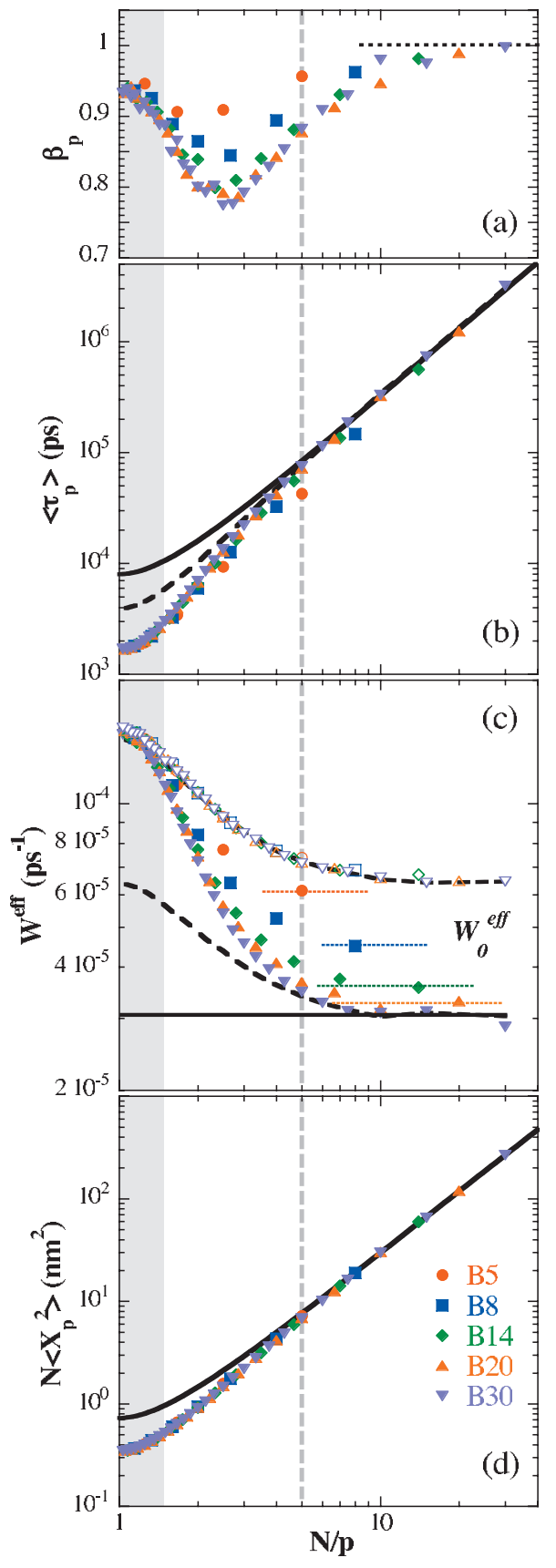

FIG. 5. Parameters from Rouse model analysis as a function of $N / p$. (a) Stretching parameters and (b) average relaxation times from KWW analysis of the Rouse correlators. (c) Rouse frequencies obtained from Eq. (14): empty symbols correspond to the simulations without uncrossability constraint. (d) Amplitudes of Rouse correlators. In all figures, shaded area represents the nonrealistic limit of the simulations, and the vertical dashed line indicates the deviations from the Rouse model at $N / p \lesssim 5$. The solid lines in (b), (c), and (d) correspond to Rouse model fits and dashed lines in (b) and (c) to ARS model fits.

collapse onto one curve when they are represented in terms of the scaling variable $N / p$. This scaling is almost fulfilled although some deviations can be envisaged, in particular for $N \leq 14$. Moreover, although in the long $N / p$ range, $\left\langle\tau_{p}\right\rangle$ nicely follows the Rouse behavior (solid line in the plot), for $N / p \lesssim 5\left\langle\tau_{p}\right\rangle$ shows strong deviations, being faster than the Rouse prediction. Interestingly, this is also the range where $\beta_{p}$-values show stronger deviations from exponentiality. From $\left\langle\tau_{p}\right\rangle$ we have calculated an effective Rouse frequency,

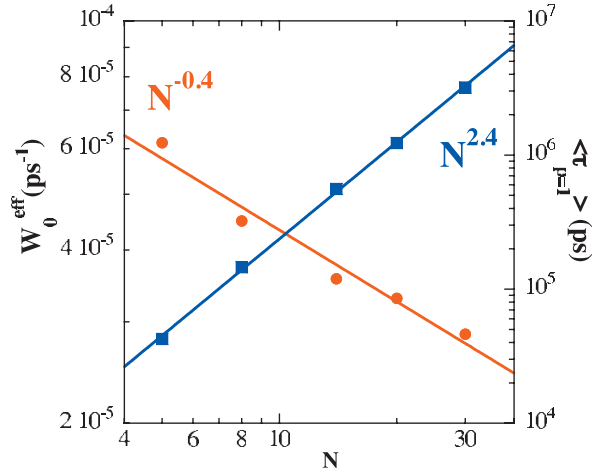

FIG. 6. $W_{0}^{\text {eff }}$ (circles) and $\left\langle\tau_{p=1}\right\rangle$ (squares) as a function of the number of blobs per chain $N$.

$W^{\text {eff }}$, by assuming Eq. (14). In this way the deviations from Rouse scaling mentioned above are highlighted as can be seen in Fig. 5(c). On the other hand, within the Rouse model the Rouse frequency should be constant. Figure 5(c) shows that this is not the case. $W^{\text {eff }}$ strongly decreases with $N / p$ reflecting the $\left\langle\tau_{p}\right\rangle$ behavior above discussed. Only for high values of $N / p, W^{\text {eff }}$ tends towards a constant plateau, $W_{0}^{\text {eff }}$. However, although for the chains with low $N$ value the plateau is not well-defined, $W_{0}^{\text {eff }}$ seems to depend on $N$. In Fig. 5(c) we have also included for comparison the results obtained for $W^{\text {eff }}$ in the case of simulations without the uncrossability algorithm. In this case, although $W^{\text {eff }}$ also depends on $N / p$ for $N / p \lesssim 8$, the values corresponding to different chain lengths collapse onto a single curve as expected from Rouselike behavior. Finally, Fig. 5(d) shows the amplitudes of the Rouse correlators $\left\langle X_{p}^{2}(0)\right\rangle$. Again we observe strong deviations from Rouse behavior [Eq. (16)] in the low $N / p$ region. In this range the amplitudes are massively suppressed indicating significantly stronger restoring forces than those originated from the entropic potential. We note that the $N / p$ region where these deviations occur is approximately the same range of $N / p$ where the deviations from Rouse behavior of both $\left\langle\tau_{p}\right\rangle$ and $\beta_{p}$ are more severe.

\section{DISCUSSION}

In Sec. IV we have mentioned that the more or less constant values of the effective Rouse rate, $W_{0}^{\text {eff }}$, for the high $N / p$ limit seem to depend on the chain length $N$. Although with high uncertainties, we have estimated such a dependence as $W_{0}^{\text {eff }} \sim N^{-0.4}$ (see Fig. 6). A plateau in $W^{\text {eff }}$ depending on $N$ is an ingredient of different models. For example, the original reptation theory predicts that the relaxation time of a Rouse mode $p$ with $N / p>N_{e}$, the entanglement length, is enhanced by a factor $3 N / N_{e}$ compared to the Rouse model. ${ }^{39,40}$ This implies that the plateau of $W^{\text {eff }}$ should decrease proportional to $N^{-1}$. If contour lengths fluctuations are included in the reptation theory, this dependence should be of the order of $N^{-1.5}$. However, in our case we found a clearly weaker dependence, which rules reptation behavior out.

The $N$-dependence found by us is closer to what was predicted by Schweizer ${ }^{44}$ in the framework of the so-called renormalized Rouse theory. This approach is based on a nonlinear generalized Langevin equation and the memory func- 


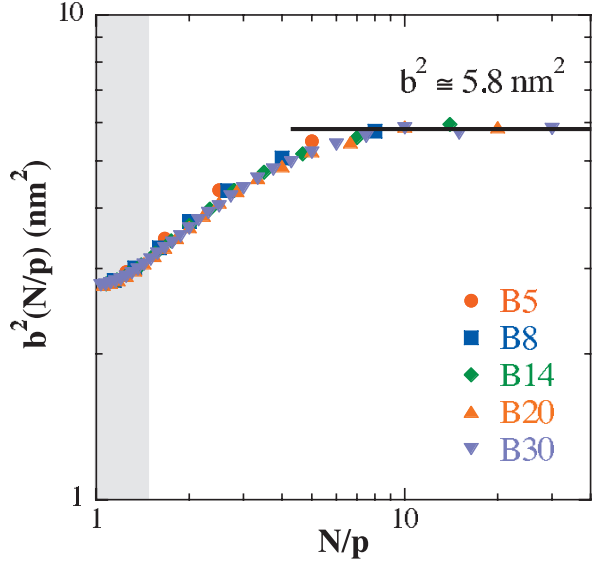

FIG. 7. $b^{2}$ as a function of the scaling variable $N / p$ for the different systems.

tion formalism. This equation involves a random force time correlation function matrix, which is responsible for an extra frictional contribution (relative to the standard Rouse description) which is both nonlocal in time and normal mode dependent. In this framework, the effective Rouse rate for the longest length scales (longest $N / p$ values) of the order of the radius of gyration results to be constant but depends on $N$ as $\sim N^{-0.4}$ as in our results.

For small and intermediate values of $N / p$ the effective Rouse rate calculated by us shows a strong $N / p$-dependence [see Fig. 5(c)], which cannot be explained in terms of the Rouse model. However, we have to take into account that, in the framework of the Rouse model, the Rouse frequency $W$ and thereby the mode relaxation times depend on both the friction coefficient $\xi$ and the restoring forces determined by the square of the statistical segment length $b^{2}$. According to Eq. (16), $b^{2}$ can be independently determined from the amplitudes of the Rouse modes shown in Fig. 5(d). The results obtained are shown in Fig. 7. As expected for well equilibrated chains, the same values of $b^{2}$ are obtained for the different chain lengths, when they are represented against the scaling variable $N / p$. However, $b^{2}$ is not constant as for an ideal chain but depends on $N / p$. Our chains are not ideal, mainly because of the angular potential which gives them some stiffness. This stiffness will have the strongest effect on the smallest length scales. Figure 7 shows that indeed for $N / p \gtrsim 7$ a constant value of $b^{2} \simeq 5.8 \mathrm{~nm}^{2}$ is obtained. As can be deduced from the values included in Table III, $b^{2} \approx\left\langle R_{e e}\right\rangle / N$ in particular for the high molecular weight chains where Gaussian statistics is better fulfilled. However, $b(\sim 2.4 \mathrm{~nm})$ results to be higher than the "bond length"average distance between blobs_of the system $(\sim 1.33 \mathrm{~nm})$.

The effect of chain stiffness on Rouse behavior can be addressed in terms of the so-called all rotational state (ARS) model. ${ }^{45}$ This model assumes that the effective statistical segment depends on $N / p$ through a $N / p$-dependence of the characteristic ratio $C(N / p)$, which only for the longest $N / p$ limit takes a constant value $C_{\infty}$. This dependence leads to a stiffness of the chain for low values of $N / p$ (local scales) and consequently the spring constant $\left(3 k_{B} T / b^{2}\right)$ increases. This is just the qualitative behavior shown by $b^{2}$ in Fig. 7. There-

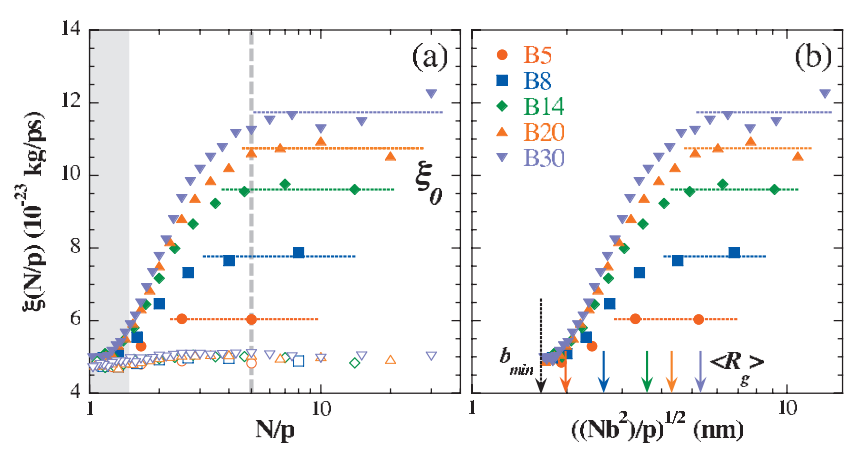

FIG. 8. (a) Effective friction $\xi$ as a function of the scaling variable $N / p$ and (b) as a function of $L=\left[N b^{2} / p\right]^{1 / 2}$. Empty symbols in (a) are the simulations without uncrossability conditions. In (b) the longest arrow corresponds to the $b_{\min }$-value and the colored arrows to the average radius of gyration of the different chains.

fore, we can see whether the local stiffness is the only reason for the deviations of $\left\langle\tau_{p}\right\rangle$ and $W^{\text {eff }}$ for the Rouse behavior in the low- $N / p$ range. To do that - and in the spirit of the ARS model-we have calculated $\left\langle\tau_{p}\right\rangle$ and $W^{\text {eff }}$ by the Rouse model [Eqs. (14) and (15)] but taking $b^{2}(N / p)$ as deduced from the amplitudes (Fig. 7). The value of the friction $\xi$ is fixed from the high $N / p$ values of $\left\langle\tau_{p}\right\rangle$ and $W^{\text {eff }}$, where Rouse-like behavior ( $W^{\text {eff }} \sim$ constant) seems to apply. The results obtained are shown for $N=30$ by dashed lines in Figs. 5(b) and 5(c). Although the agreement is better than in the case of pure Rouse, it is obvious that local stiffness cannot be the only reason for the low $-N / p$ behavior. Interestingly, a Rouse model corrected for the chain stiffness in the same way gives an almost perfect description of the $W^{\text {eff }}$ behavior obtained from the simulations without the uncrossability algorithm [see Fig. 5(c)]. On the other hand, the $N$-dependence of $W_{0}^{\text {eff }}$, above described, cannot be easily explained in terms of local stiffness either.

These results suggest to postulate a mode dependent friction as an additional mechanism. The values of a mode dependent friction coefficient for the different chain lengths can be calculated as

$$
\xi(N / p)=\frac{3 k_{B} T}{W^{\mathrm{eff}} b^{2}},
$$

where $W^{\text {eff }}$ and $b^{2}$ depend on $N / p$ [Figs. 5(c) and 7]. The results obtained are shown in Fig. 8(a). The effective friction coefficient so obtained strongly increases at low values of $N / p$ reaching a constant plateau $\xi_{0}$, which clearly depends on $N$ as it is shown in Fig. 9 (circles). Obviously, the $N$-dependence of $\xi_{0}$ is similar to that found for $W_{0}^{\text {eff }}$ because in the high $N / p$-range $b^{2} \sim$ constant. A $N$-dependence for the friction coefficient calculated from both, the Rouse time $\left(\tau_{1}\right)$ and the diffusion coefficient (i.e., at large scales $N / p$ ), in simulated polyethylene melts has also been reported (see Fig. 9 of Ref. 7). It is worthy of remark that in that case the simulation method was fully atomistic MD, i.e., the $\mathrm{N}$-dependence of the friction coefficient seems to be independent of the simulation method. We have also estimated the values of the chain-diffusion coefficient, $D$, from the long-time limit of the mean square displacement of the chain center of mass. From the results obtained we have deduced 


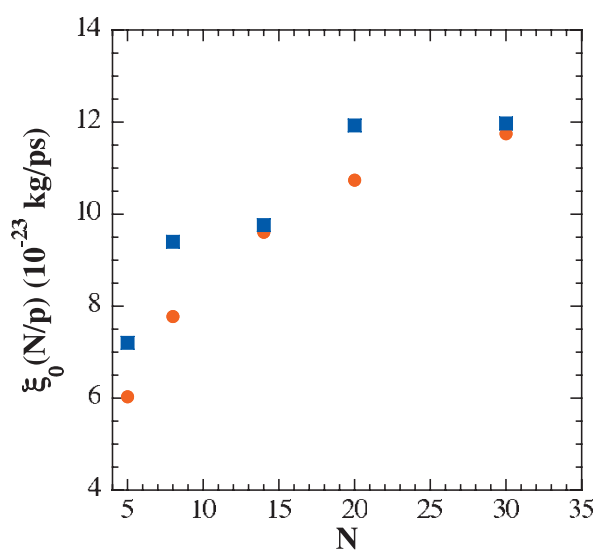

FIG. 9. Effective friction $\xi_{0}$ as a function of the number of blobs per chain $N$ calculated from the plateau of $\xi(N / p)$ at large $N / p$ (circles) and from the diffusion coefficient (squares).

the corresponding friction coefficient values as $\xi$ $=k_{B} T(D N)^{-1}$. They are also included in Fig. 9 for comparison. They show the same trend within the higher uncertainty involved in this calculation. However, a tendency to saturate at $N \rightarrow \infty$ cannot be ruled out as in the case of the polyethylene results above mentioned.

Figure 8(a) also shows for comparison the results obtained from simulations carried out without the uncrossability algorithm. As expected from the corresponding results of $W^{\text {eff }}$ described in Fig. 5(c), the friction coefficient in this case is almost constant indicating a Rouse-like behavior, which could be expected from the structure of the Langevin equation [Eq. (2)]. This almost constant value is rather close to that deduced from the bare friction frequency $(\zeta / M$ $=36.0 \mathrm{ps}^{-1}$ ) imposed in the Langevin equation to match the time scale of the atomistic simulations (see Sec. II D). Taking into account the blob mass $(0.7 \mathrm{~kg} / \mathrm{mol})$ we deduced a bare friction coefficient value of $\zeta=4.2 \times 10^{-23} \mathrm{~kg} \mathrm{ps}^{-1}$. On the other hand, in Fig. 8(b) we have displayed the "lengthscale" dependence of the effective friction coefficient. The $x$-axis of this figure represents the length scale associated to the wavelength $N / p$ and calculated as $L=\left[N b^{2} / p\right]^{1 / 2}$. This length scale has been calculated by considering the $b^{2}(N / p)$ shown in Fig. 7. The arrows also mark the values of the radius of gyration $\left\langle R_{g}\right\rangle$ of the different systems simulated (see Table III) and the minimum $b$-value (see Fig. 7) as well. Interestingly, the effective friction for a given chain length $N$ is constant in both limits $L \sim b_{\min }$ and $L \gtrsim\left\langle R_{g}\right\rangle$ and strongly increases for intermediate length scales. Moreover, the values for $L \sim b_{\min }$ are for all chains rather similar to those obtained from the simulations without the uncrossability algorithm. Thereby it seems that the increase in the effective friction at larger length scales is mainly due to additional molecular (blob) interactions-other than those included in the potential of mean force. These interactions are accounted for in the model by means of the uncrossability algorithm. Although these effects are even present in the case of short chains, obviously they become more important as soon as the chain length increases (see Fig. 8).

The length-scale regime where the effective friction increases is also the range where the Rouse correlators display

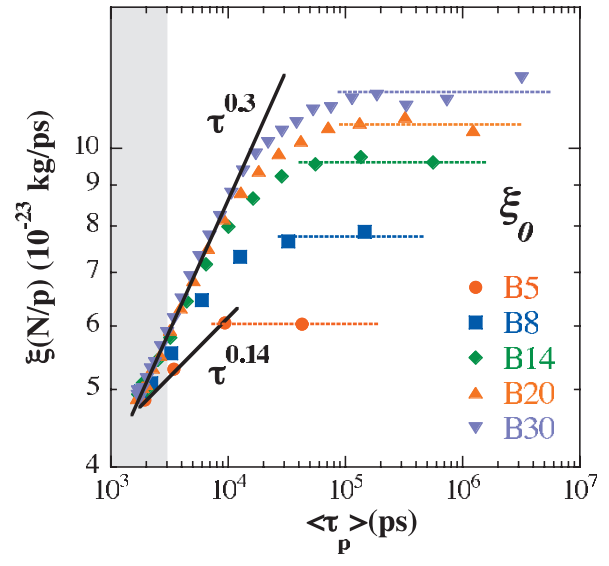

FIG. 10. Effective friction $\xi$ as a function of the average relaxation times $\left\langle\tau_{p}\right\rangle$ for the different systems.

strong nonexponentiality. The nonexponential behavior of the Rouse correlators is a consequence of time correlation among the forces acting upon a chain bead, ${ }^{14}$ as is assumed in the memory function formalisms of the generalized Langevin equation and in particular in the renormalized Rouse model mentioned above. In this scenario, we should expect a time-dependent friction. We can have an idea about this time dependence by taking into account the representative time scale $\left\langle\tau_{p}\right\rangle$ corresponding to a wavelength $N / p$. The results so obtained are shown in Fig. 10.

The effective friction coefficient increases dramatically with time at the intermediate time scales. This behavior can be approximated by a $t^{\gamma}$-dependence where the $\gamma$-values depend on $N$. If this "time dependence" also reflects the correlation between forces, both $\gamma$ and the nonexponential parameter $\beta$-values should be related. Indeed this is the case as it is shown in Fig. 11, where $\gamma$-values are represented as a function of $1-\beta_{\min }$, being $\beta_{\min }$ the minimum value of $\beta_{p}$ corresponding to a given chain length $N$. Figure 11 shows that a $\gamma \simeq 1-\beta_{\min }$ correlation is rather well fulfilled.

It is worth to mention that the behavior obtained for the effective friction coefficient (Figs. 8 and 9) as well as the correlation found between $\gamma$ and $\beta_{p}$ (Fig. 11) are-at least qualitatively - in agreement with the prediction for renormalized Rouse models in general ${ }^{46}$ and in particular for the

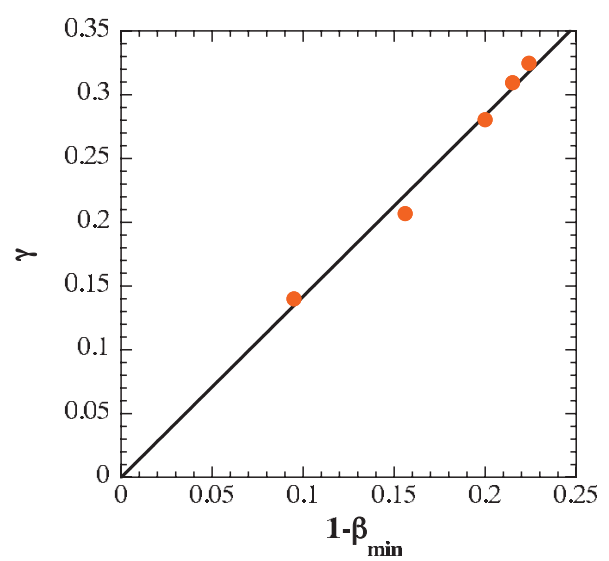

FIG. 11. Correlation between the $\gamma$ parameter from the $t^{\gamma}$-dependence of the friction coefficient and the $\beta_{\mathrm{min}^{-}}$-values. 


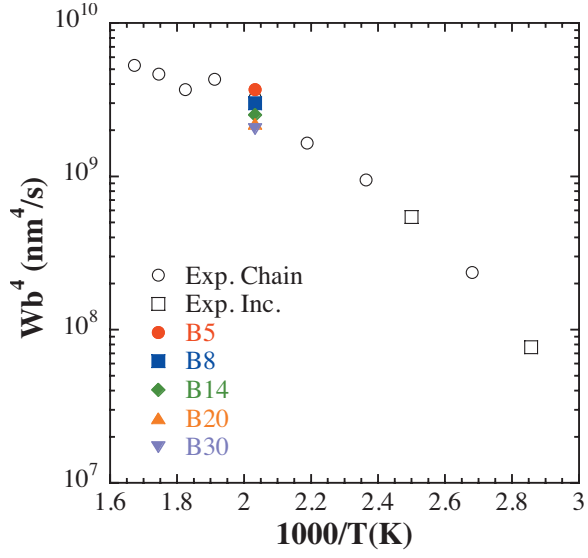

FIG. 12. Rouse variable $W b^{4}$ as a function of temperature from the simulation results calculated as the large $N / p$-limit of $W_{\text {eff }} b^{4}$ (full symbols) and from the short-time regime of the single chain dynamic structure factor published in Refs. 1 and 49 for a PEP sample of $M_{w} \simeq 80000 \mathrm{~g} / \mathrm{mol}$ (empty circles). The two low temperature values (empty squares) are estimated from the incoherent neutron scattering results on a PEP sample of $M_{w}$ $\simeq 20000 \mathrm{~g} / \mathrm{mol}$ (Ref. 36).

simple theoretical approach proposed by Schweizer. ${ }^{44}$ On the other hand, we do not observe signatures of reptation at the $N$-values here investigated $N \leq 6 N_{e}$. This is in agreement with the viscoelastic and diffusion measurements reported in Ref. 47. In that paper it was shown that the normalized product of viscosity and self-diffusion coefficient was at $373 \mathrm{~K}$ almost constant for PEP samples with $M_{w} / M_{e}<6$. It is worthy of remark that this result is in principle in agreement not only with simple Rouse model predictions but also with those from the renormalized Rouse model. ${ }^{44}$ In contrast, reptation theory predicts an increasing function of $N\left(M_{w}\right)$. Finally, it would be interesting to check the applicability of the coarse-grained model here developed for describing real PEP behavior. To do that, the right magnitude to be considered is $b^{2} / \xi_{\text {eff }}$ because it should be independent of the level of coarse graining (see Sec. IV). In fact, the so-called Rouse variable $W b^{4}$, which can be determined by neutron scattering experiments, is just proportional to $b^{2} / \xi_{\text {eff }}\left[W b^{4}=3 k_{B} T b^{2} \xi^{-1}\right.$ (Ref. 1)]. Chain dynamics of PEP have been investigated by neutron scattering mainly in the high molecular weight range $M_{w} / M_{e}>20$, where clear evidences of reptation behavior are found. ${ }^{48,49}$ However, the Rouse variable $W b^{4}$ can be obtained from the short-time regime of the single chain dynamic structure factor ${ }^{1}$ even in the case of reptation. This magnitude should hardly depend on the molecular weight. The values reported ${ }^{1,49}$ for $W b^{4}$ of PEP at different temperatures are shown in Fig. 12. This figure also includes two low temperature values of $W b^{4}$ estimated from incoherent neutron scattering results on a PEP sample of $M_{w} \simeq 20000 \mathrm{~g} / \mathrm{mol}^{36}$ From the simulation results reported here, we can also calculate $W b^{4}$ as the large $N / p$-limit of $W_{\text {eff }} b^{4}$. In that regime both $W_{\text {eff }}\left(W_{0}^{\text {eff }}\right)$ and $b$ are basically constant. The values so obtained are also included in Fig. 12. As $W_{0}^{\text {eff }} \sim N^{-0.4}$, the values estimated of $W b^{4}$ slightly change with $N\left(M_{w}\right)$. However, they perfectly agree in the average with the experimental value deduced from neutron scattering measurements at $492 \mathrm{~K}$.

In any case, a direct comparison of the single chain dy-

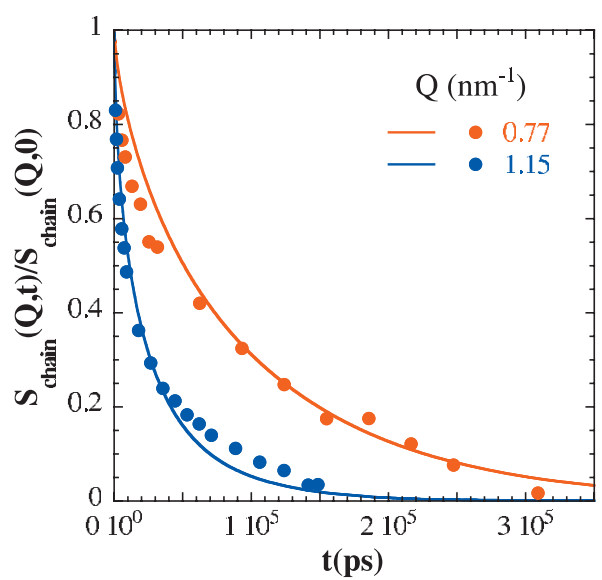

FIG. 13. Time dependence of the normalized single chain dynamic structure factor calculated from the coarse-grained simulations of B8 $\left(M_{w}\right.$ $=5600 \mathrm{~g} / \mathrm{mol}$ ) (lines) and measured by neutron scattering experiments $\left(M_{w} \simeq 6000 \mathrm{~g} / \mathrm{mol}\right)$ (points).

namic structure factor calculated from the coarse-grained simulations and measured by neutron scattering on samples with the same molecular weight would be desirable. Figure 13 displays some preliminary results in this direction. This figure shows the normalized single chain dynamic structure factor $S_{\text {chain }}(Q, t) / S_{\text {chain }}(Q, 0)$ at two low $Q$-values measured on a PEP sample with $M_{w} \simeq 6000 \mathrm{~g} / \mathrm{mol}$ in comparison with the same magnitude calculated from the coarse-grained simulations of B8 $\left(M_{w}=5600 \mathrm{~g} / \mathrm{mol}\right)$ sample. Data are represented in a linear time scale to emphasize the long time behavior. The agreement found seems to be rather good.

\section{SUMMARY AND CONCLUSIONS}

In this work we have constructed a coarse-grained model of PEP starting from fully atomistic MD simulation results of a PEP cell containing 12 chains of 80 monomers each $\left(M_{w}\right.$ $=5600 \mathrm{~g} / \mathrm{mol})$. In this way we were able to extend our simulations to higher molecular weights $\left(M_{w} \sim 6 M_{e}\right)$ and longer times. We want to stress that in our approach we started from the bottom, i.e., all coarse-grained parameters were determined from the atomistic simulation runs, which were previously validated by extensive neutron scattering measurements. Moreover, the results from coarse-grained simulations are in good agreement with experimental results available.

For all molecular weights (chain lengths) investigated, we observe deviations from Rouse behavior even when chain stiffness is considered in the Rouse model. These deviations become more evident as the length chain increases. We have shown that this behavior can be rationalized in terms of a mode-number (length-scale) dependent friction coefficient. The increase in this effective friction at the intermediate scales $\left(b_{\min } \leq L \leq R_{g}\right)$ seems to be due to blob interactions other than those included in the intermolecular soft potential of mean force. They are accounted for in the model by means of the uncrossability algorithm. These interactions are at the end the reason for correlation among the forces acting upon a chain bead and thereby for the nonexponentiality of the Rouse correlators. However, it seems that in the molecular weight range here explored $\left(M_{w} \lesssim 6 M_{e}\right)$ these effects still 
give not raise to reptation behavior but to a crossover regime between Rouse and reptation that can be understood, at least qualitatively, in terms of the so-called generalized Rouse models.

\section{ACKNOWLEDGMENTS}

The authors acknowledge support of the European Community within the SoftComp Network of Excellence (NoE) program.

We thank support by the "Donostia International Physics Center," the European Commission NoE SoftComp, Contract No. NMP3-CT-2004-502235, the projects MAT2007-63681, IT-436-07 (GV), and the Spanish Ministerio de Educacion y Ciencia (Grant No. CSD2006-53). R.P.A. acknowledges the Grant No. BES-2005-10794 (MAT2004-01017).

${ }^{1}$ D. Richter, M. Monkenbusch, A. Arbe, and J. Colmenero, Neutron Spin Echo in Polymer Systems, Advances in Polymer Science (SpringerVerlag, Berlin, 2005) Vol. 174.

${ }^{2}$ A. Narros, A. Arbe, F. Alvarez, J. Colmenero, and D. Richter, J. Chem. Phys. 128, 224905 (2008).

${ }^{3}$ A.-C. Genix, A. Arbe, F. Alvarez, J. Colmenero, L. Willner, and D. Richter, Phys. Rev. E 72, 031808 (2005).

${ }^{4}$ O. Ahumada, D. N. Theodorou, A. Triolo, V. Arrighi, C. Karatasos, and J.-P. Ryckaert, Macromolecules 35, 7110 (2002).

${ }^{5}$ W. Paul, G. D. Smith, and D. Y. Yoon, Macromolecules 30, 7772 (1997).

${ }^{6}$ W. Paul, G. D. Smith, D. Y. Yoon, B. Farago, S. Rathegeber, A. Zirkel, L. Willner, and D. Richter, Phys. Rev. Lett. 80, 2346 (1998).

${ }^{7}$ V. A. Harmandaris, V. G. Mavrantzas, and D. N. Theodorou, Macromolecules 31, 7934 (1998); 33, 8062 (2000).

${ }^{8}$ M. Brodeck, F. Alvarez, A. Arbe, F. Juranyi, T. Unruh, O. Holderer, J. Colmenero, and D. Richter, J. Chem. Phys. 130, 094908 (2009).

${ }^{9}$ J. T. Padding and W. J. Briels, J. Chem. Phys. 114, 8685 (2001).

${ }^{10} \mathrm{~K}$. Binder, Monte Carlo and Molecular Dynamics Simulations in Polymer Science (Oxford University Press, New York, 1995).

${ }^{11}$ K. Kremer, G. S. Grest, and I. Carmesin, Phys. Rev. Lett. 61, 566 (1988).

${ }^{12}$ M. Kröger and S. Hess, Phys. Rev. Lett. 85, 1128 (2000).

${ }^{13}$ J. Baschnagel and F. Varnik, J. Phys.: Condens. Matter 17, R851 (2005).

${ }^{14}$ A. J. Moreno and J. Colmenero, Phys. Rev. Lett. 100, 126001 (2008).

${ }^{15}$ K. Kremer and T. Müller-Plathe, MRS Bull. 26, 205 (2001).

${ }^{16}$ J. Baschnagel, K. Binder, P. Doruker, A. A. Gusev, O. Hahn, K. Kremer, W. L. Mattice, F. Müller-Plathe, M. Murat, W. Paul, S. Santos, U. W. Suter, and V. Tries, Bridging the Gap Between Atomistic and CoarseGrained Models of Polymers: Status and Perspectives, Advances in Polymer Science (Springer-Verlag, Berlin, 2000), Vol. 152.

${ }^{17}$ V. A. Harmandaris, N. P. Adhikari, N. F. A. van der Vegt, and K. Kremer,
Macromolecules 39, 6708 (2006).

${ }^{18}$ P. Carbone, H. A. K. Varzaneh, X. Chen, and F. Müller-Plathe, J. Chem. Phys. 128, 064904 (2008)

${ }^{19}$ C. Chen, P. Depa, J. Maranas, and V. Garcia Sakai, J. Chem. Phys. 128, 124906 (2008).

${ }^{20}$ P. K. Depa and J. K. Maranas, J. Chem. Phys. 126, 054903 (2007).

${ }^{21}$ S. J. Marrink, H. J. Risselada, S. Yefimov, D. P. Tieleman, and A. H. de Vries, J. Phys. Chem. B 111, 7812 (2007).

${ }^{22}$ V. A. Harmandaris and K. Kremer, Macromolecules 42, 791 (2009).

${ }^{23}$ R. L. C. Akkermans and W. J. Briels, J. Chem. Phys. 113, 6409 (2000); 114, 1020 (2001).

${ }^{24}$ J. T. Padding and W. J. Briels, J. Chem. Phys. 115, 2846 (2001).

${ }^{25}$ J. T. Padding and W. J. Briels, J. Chem. Phys. 117, 925 (2002).

${ }^{26}$ P. J. Hoogerbrugge and J. M. V. A. Koelman, Europhys. Lett. 19, 155 (1992).

${ }^{27}$ L. J. Fetters, D. J. Lohse, and R. H. Colby, in Physical Properties of Polymers Handbook, 2nd ed., edited by J. E. Mark (Springer, New York, 2007) Chap. 25.

${ }^{28}$ H. Sun and D. Rigby, Spectrochim. Acta A153, 1301 (1997).

${ }^{29}$ D. Rigby, H. Sun, and B. E. Eichinger, Polym. Int. 44, 311 (1997).

${ }^{30}$ H. Sun, J. Phys. Chem. B 102, 7338 (1998).

${ }^{31}$ H. Sun, P. Ren, and J. R. Fried, Comput. Theor. Polym. Sci. 8, 229 (1998).

${ }^{32}$ S. W. Bunte and H. Sun, J. Phys. Chem. B 104, 2477 (2000).

${ }^{33}$ J. Yang, Y. Ren, A. Tian, and H. Sun, J. Phys. Chem. B 104, 4951 (2000).

${ }^{34}$ M. J. McQuaid, H. Sun, and D. Rigby, J. Comput. Chem. 25, 61 (2004).

${ }^{35}$ D. N. Theodorou and U. W. Suter, Macromolecules 18, 1467 (1985).

${ }^{36}$ R. Pérez-Aparicio, A. Arbe, F. Alvarez, J. Colmenero, and L. Willner, Macromolecules 42, 8271 (2009).

${ }^{37}$ K. Kremer and G. S. Grest, J. Chem. Phys. 92, 5057 (1990).

${ }^{38}$ M. P. Allen, Mol. Phys. 40, 1073 (1980); 47, 599 (1982).

${ }^{39}$ P. E. Rouse, J. Chem. Phys. 21, 1272 (1953).

${ }^{40}$ M. Doi and S. F. Edwards, The Theory of Polymer Dynamics (Oxford University, Oxford, England, 1986).

${ }^{41}$ J. S. Shaffer, J. Chem. Phys. 103, 761 (1995).

${ }^{42}$ G. D. Smith, W. Paul, M. Monkenbusch, and D. Richter, Chem. Phys. 261, 61 (2000)

${ }^{43}$ M. Guenza, Phys. Rev. Lett. 88, 025901 (2001).

${ }^{44}$ K. S. Schweizer, J. Chem. Phys. 91, 5802 (1989).

${ }^{45}$ S. Brückner, Macromolecules 14, 449 (1981).

${ }^{46} \mathrm{R}$. Kimmich and N. Fatkullin, Polymer Chain Dynamics and NMR, Advances in Polymer Science, Advances in Polymer Science (SpringerVerlag, Berlin, 2004), Vol. 170.

${ }^{47}$ C. B. Gell, W. W. Graessley, and L. J. Fetters, J. Polym. Sci. 35, 1933 (1997).

${ }^{48}$ D. Richter, B. Farago, L. J. Fetters, J. S. Huang, B. Ewen, and C. Lartigue, Phys. Rev. Lett. 64, 1389 (1990).

${ }^{49}$ D. Richter, B. Farago, R. Butera, L. J. Fetters, J. S. Huang, and B. Ewen, Macromolecules 26, 795 (1993). 